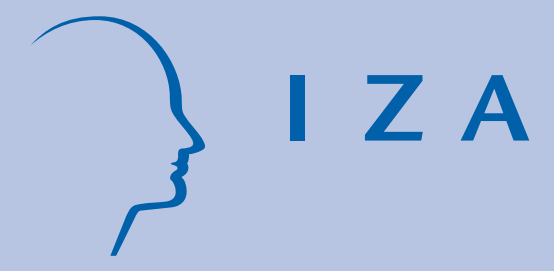

IZA Standpunkte Nr. 64

Beschäftigungsfähigkeit als ein zentraler Faktor eines längeren Erwerbslebens

Werner Eichhorst

Februar 2014

Forschungsinstitut zur Zukunft der Arbeit Institute for the Study of Labor 


\title{
Beschäftigungsfähigkeit als ein zentraler Faktor eines längeren Erwerbslebens
}

\author{
Werner Eichhorst \\ IZA
}

\author{
IZA Standpunkte Nr. 64 \\ Februar 2014
}

IZA

Postfach 7240

53072 Bonn

Tel.: (0228) 3894-0

Fax: (0228) 3894-180

E-Mail: iza@iza.org

Die Schriftenreihe „IZA Standpunkte” veröffentlicht politikrelevante Forschungsarbeiten und Diskussionsbeiträge von IZA-Wissenschaftlern, IZA Research Fellows und IZA Research Affiliates in deutscher Sprache. Die Autoren sind für den Inhalt der publizierten Arbeiten verantwortlich. Im Interesse einer einheitlichen Textzirkulation werden Aktualisierungen einmal publizierter Arbeiten nicht an dieser Stelle vorgenommen, sondern sind gegebenenfalls nur über die Autoren selbst erhältlich. 
IZA Standpunkte Nr. 64

Februar 2014

\section{ZUSAMMENFASSUNG}

\section{Beschäftigungsfähigkeit als ein zentraler Faktor eines längeren Erwerbslebens ${ }^{1}$}

Angesichts des demographischen Wandels wird es immer wichtiger, dass sich Unternehmen und Erwerbstätige auf ein längeres Erwerbsleben einstellen. Eine höhere Beschäftigungsquote älterer Arbeitskräfte und ein späterer Renteneintritt erlauben die Mobilisierung von dringend benötigten Arbeitskräfte- und Fachkräftepotenzialen. Dies wird um so besser gelingen, je mehr auf Pflege und Entwicklung der Beschäftigungsfähigkeit im Lebensverlauf geachtet wird, sowohl durch Aus- und Weiterbildung als auch durch die Gestaltung von Arbeitsbedingungen und Entwicklungsmöglichkeiten, die ein längeres, gesünderes und produktiveres Erwerbsleben erlauben. Im nachfolgenden Text sollen die wesentlichen Herausforderungen aufgezeigt und Lösungsansätze vorgestellt werden, die Politik, Sozialpartner, Betriebe und Beschäftigte gleichermaßen angehen.

JEL-Codes: J11, J14, J26, J28

Schlagworte: ageing, demographic trends, elderly, labor force projection, public policy, retirement, continuous vocational training, working conditions

Kontaktadresse:

Werner Eichhorst

IZA

Postfach 7240

D-53072 Bonn

E-Mail: eichhorst@iza.org

1 Überarbeitete Fassung eines Vortrags beim DASA-Symposium 2013 „Altern ist Zukunft!“ in Dortmund am 8. November 2013. 


\section{Einleitung: Der demographische Wandel ist eine fundamentale Veränderung}

Für die künftige Entwicklung des Arbeitsmarktes in Deutschland sowie in weiteren Zusammenhängen auch in Europa und weltweit stellen der demographische Wandel und die damit einhergehenden Veränderungen der Erwerbsbevölkerung und ihrer Altersstruktur neben der fortschreitenden Globalisierung und technologischen Innovationen - eine zentrale Herausforderung dar. Damit wird auch die Entwicklung und Pflege der Beschäftigungsfähigkeit von Erwerbspersonen (und indirekt auch von Unternehmen) eines der Themen sein, die in den nächsten Jahren noch an Bedeutung gewinnen werden. Vielen europäischen Staaten und gerade auch Deutschland steht eine Situation bevor, in der Arbeitskräfte deutlich knapper werden und das Durchschnittsalter der Erwerbstätigen massiv ansteigen wird. Das bedeutet aus Sicht der Personalrekrutierung auch, dass es wesentlich weniger junge Nachwuchskräfte geben wird, die über eine duale Berufsausbildung verfügen oder als Universitäts- oder Fachhochschulabsolventen für die Unternehmen gewonnen werden können. Jedes einzelne Unternehmen, aber auch die Gesellschaft insgesamt wird sich künftig mehr noch als in der Vergangenheit mit bestimmten Potenzialen, mit bestimmten Gruppen und bestimmten Problemlagen auseinandersetzen und diese zu mobilisieren versuchen, die in den letzten 10 oder 20 Jahren nicht so sehr im Fokus standen und mehr oder weniger klar vernachlässigt worden sind. Dabei sind insbesondere die Potenziale weiblicher Erwerbspersonen zu beachten. Die Frage, was in diesem Bereich zu erreichen ist, ist eines der wichtigen Themen mit dem sich das Forschungsinstitut zur Zukunft der Arbeit beschäftigt hat. Gleiches gilt für ein zweites zentrales Thema wie die Verlängerung des Erwerbslebens und einem damit verbundenen späteren Renteneintritt. Auch hier haben sich politische Rahmenbedingungen in den letzten Jahren deutlich geändert. Dies hat bereits unmittelbare Implikationen auf der Ebene der Unternehmen, aber auch der Individuen gezeigt. Drittens ist auch das große Thema der Migration und Integration als zusätzliches Handlungsfeld zu beachten. Ein Handlungsfeld, das zunehmend sowohl in Deutschland als auch in vielen anderen europäischen Staaten an Bedeutung gewinnen wird, um mit den Konsequenzen des demographischen Wandels und der Verknappung des Erwerbsangebots besser zurechtkommen zu können.

Deutschland spürt bereits jetzt die Auswirkung der demographischen Veränderung auf den Arbeitsmarkt und die Sozialsysteme. Tatsächlich steht aber in absehbarer Zukunft eine weit darüber hinausgehende Verknappung der verfügbaren Erwerbspersonen bevor. Im 
Wesentlichen wird dies zum Ende dieser Dekade und dann in den 2020er Jahren massiv zu Buche schlagen. Im Moment gibt es in Deutschland etwa 42 Millionen Erwerbstätige und es werden nach allen vorliegenden Prognosen etwa 6 Millionen in den nächsten 15 Jahren wegfallen. Das sind etwa 15 Prozent. Es kann dann also binnen eines Jahrzehnts etwa jeder siebte derzeitig Beschäftigte nicht mehr ohne weiteres adäquat ersetzt werden. Es besteht deshalb die klare Notwendigkeit, die uns zur Verfügung stehenden Ressourcen und Potenziale wesentlich intensiver $\mathrm{zu}$ nutzen und $\mathrm{zu}$ mobilisieren. Das hat massive Veränderungen in der Politik, in der Personalpolitik und auch für die Individuen zur Folge. Nicht überall sind die Zeichen der Zeit erkannt worden, und umso wichtiger ist es, auf die Notwendigkeit des Wandels in Politik, Arbeitswelt und individueller Lebensplanung hinzuweisen - und Beschäftigungsfähigkeit kann dabei das Leitmotiv sein, um den Unternehmen qualifizierte und produktive Arbeitskräfte zu sichern und gleichzeitig im individuellen Lebensverlauf erwerbstätig sein zu können, um damit Einkommen, Altersvorsorge und gesellschaftliche Integration zu erreichen. 


\section{$\underline{\text { Handlungsfelder und Potenziale }}$}

Eine Expertenkommission der Robert Bosch Stiftung, die sich mit dem Thema „Arbeitswelt 2030" beschäftigt hat, hat in ihrem 2013 erschienenen Bericht drei zentrale Handlungsfelder für die erfolgreiche Bewältigung des demographischen Wandels aufgezeigt und im Hinblick auf die Konsequenzen für die Politik, die Unternehmen und jeden Einzelnen diskutiert: ${ }^{2}$

1) „Köpfe“: Wohnbevölkerung/Erwerbsbevölkerung

2) „Zeit“: Arbeitsvolumen/Erwerbstätigkeit

3) „Produktivität“: Bildung/Qualifikation/Innovation

Zum ersten Punkt muss die wichtige Frage, was durch Migration erreicht werden kann, beantwortet werden. Hier hat Deutschland zwar in den letzten Jahren deutlich an Attraktivität gewonnen, was die gewachsenen Netto-Migrationssalden belegen, jedoch ist hier keine leichte Prognose möglich, und die weitere Entwicklung hängt von der relativen Attraktivität Deutschlands als Zielland für Zuwanderung ab. Das möglicherweise größere und leichter zu mobilisierende Potenzial liegt vermutlich eher im zweiten Segment, also bei der Arbeitszeit und bei der Erwerbstätigkeit. Als drittes Handlungsfeld muss die Produktivität, also das Ergebnis von effizienter Arbeitsorganisation, technologischem Fortschritt sowie Ausund Weiterbildung und Innovationen betrachtet werden, um die vorhandenen Ressourcen, gerade die Personalressourcen wesentlich besser nutzen zu können. Das führt dann letztlich wieder auf den Punkt der Beschäftigungsfähigkeit, denn nur wer beschäftigungsfähig ist, sowohl als Arbeitgeber als auch als Erwerbstätiger, kann entsprechend stärker in den Arbeitsmarkt eingebunden werden bzw. entsprechend Arbeitsplätze schaffen und erhalten, mit denen man in Situationen eines verschärften demographischen Wandels produktiv sein kann. Wenn man sich die derzeit erwerbstätige Bevölkerung nach ihrem Erwerbsstatus in Deutschland anschaut, stellt man fest, dass es auf jeden Fall noch großes Potential im Bereich der Erwerbstätigkeit gibt. Die Beschäftigungsquoten insbesondere im höheren Lebensalter und bei Frauen könnten deutlich höher sein. Viele Frauen sind zwar erwerbstätig, jedoch nur Teilzeit. Der Teilzeitanteil bei Frauen ist in Deutschland unverhältnismäßig groß, und viele Frauen, die derzeit in Teilzeit oder Minijob arbeiten, arbeiten weit unterhalb ihrer Qualifikationen und ihrer gewünschten Arbeitszeiten. Das birgt ein erhebliches zusätzliches Arbeits- und Fachkräftepotenzial.

${ }^{2}$ Klös et al. (2013) 
Zwar hat die Erwerbstätigkeit älterer Arbeitskräfte in Folge veränderter renten- und arbeitsmarktpolitischer Bedingungen bereits in Deutschland stark zugenommen. Dennoch nimmt nach wie vor im höheren Lebensalter, bereits vor dem gesetzlichen Renteneintrittsalter, die Erwerbstätigkeit deutlich ab. So befinden sich viele Personen entweder in den Sozialleistungen oder stehen dem Erwerbsprozess letztendlich gar nicht zur Verfügung. Zusammenfassend gibt es zwei wesentliche Potenziale: das weibliche und das der älteren Arbeitskräfte. Bei den Frauen bedeutet dies insbesondere die Verlängerung der Arbeitszeiten aus dem Teilzeitbereich in den Vollzeitbereich hinein.

In der erwähnten Studie für die Bosch-Stiftung sind diese Potenziale durchgerechnet worden, um herauszufinden, welche zusätzlichen Erwerbspersonen und Arbeitsvolumina mobilisiert werden müssten. Dies ist zunächst eine rein quantitative Betrachtung dessen, was an zusätzlicher Erwerbstätigkeit und Arbeitszeit durch geänderte Bedingungen im politischen Raum und auch in den Unternehmen mobilisiert werden kann. Ausgehend vom Status quo steht ein massiver Rückgang der Erwerbstätigen bis 2030 bevor, in der Größe von etwa 6 Millionen Erwerbstätigen, die verloren gehen. Jedoch können die einzelnen Stellgrößen relativ stark durch arbeitsmarkt- und sozialpolitische sowie unternehmerische und individuelle Entscheidungen beeinflusst werden. Hierbei ist der Renteneintritt als ganz wichtige Größenordnung zu nennen, was auch wieder sehr viel mit Beschäftigungsfähigkeit im höheren Lebensalter zu tun hat. Auch die Erhöhung der Erwerbsquote der über 60jährigen auf etwa das Niveau der Personen zwischen 55 und 60 Jahren, würde eine erhebliche Zahl von möglichen Erwerbstätigen aktivieren. Hierbei sind nicht Alterskohorten gemeint, die kurz vor dem Renteneintritt stehen. In einem weiteren Szenario wurde abgeschätzt, wie sich eine Halbierung der Differenz der Erwerbsquote der Frauen zu der der Männer auswirken würde. Das alles zusammen bietet erhebliches Potential um die verfügbaren Köpfe, die erwerbstätig sein könnten, in Deutschland bis 2030 etwa auf dem Niveau zu stabilisieren, das wir derzeit haben. 
Abbildung 1: Übersicht der vorausberechneten Erwerbspersonenzahl

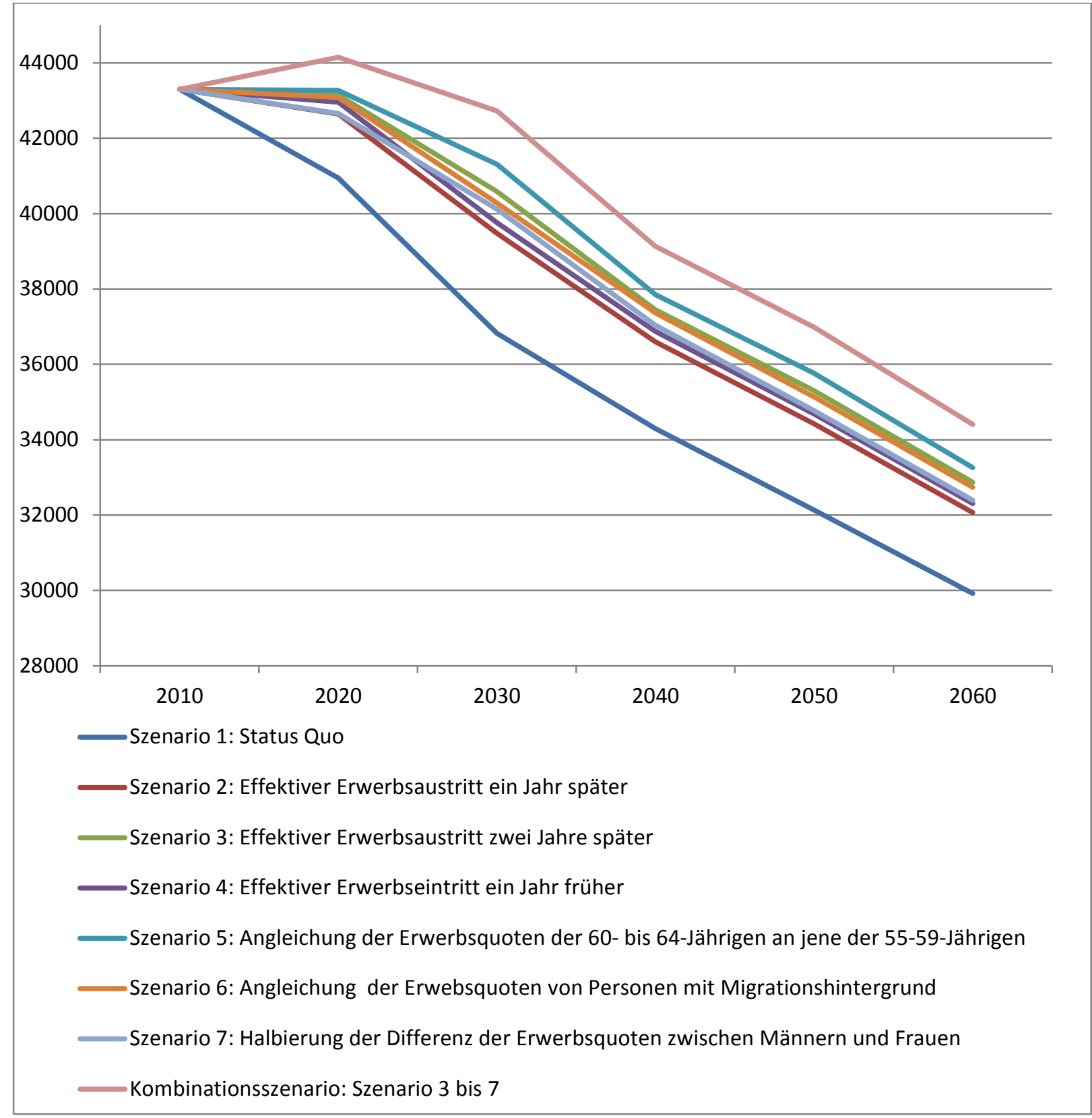

Quelle: Ehing und Moog (2013)

Zusätzlich wurden auch Berechnungen angestellt, die zeigen, dass eine Anhebung der Arbeitszeiten aus den Teilzeitbereichen in den Vollzeitbereich hinein ein beachtliches zusätzliches Potenzial bietet - und zwar ohne eine Verlängerung der Arbeitszeiten im Vollzeitbereich, bei dem Deutschland bereits jetzt vergleichsweise lange effektive Wochenarbeitszeiten aufweist. Im Gegensatz zur Teilzeit, bei der im Mittel nur geringe Stundenzahlen erreicht werden. Wenn ein Drittel der Frauen und Männer, die derzeit Teilzeit arbeiten, in Vollzeitbeschäftigung gebracht werden würden, wäre es sogar möglich, die verfügbaren Arbeitsvolumina bis 2030 zu vergrößern. Um das zu sichern muss natürlich einiges erreicht werden, vor allem in dem Bereich der derzeitigen Anreize, Teilzeitarbeit zu 
verrichten (Minijobs und Ehegattensplitting) und natürlich auch im Bereich Matching zwischen Qualifikation und Arbeitsplätzen. Ein kleiner Wehmutstropfen wird sein, dass nach 2030 die Situation noch einmal ein Stück schwieriger wird, weil dann eben diese Potenziale schon mobilisiert sein werden, während die demographische Veränderung dann noch einmal an Schärfe gewinnen wird.

Abbildung 2: Übersicht der Arbeitszeitszenarien: Arbeitsvolumen

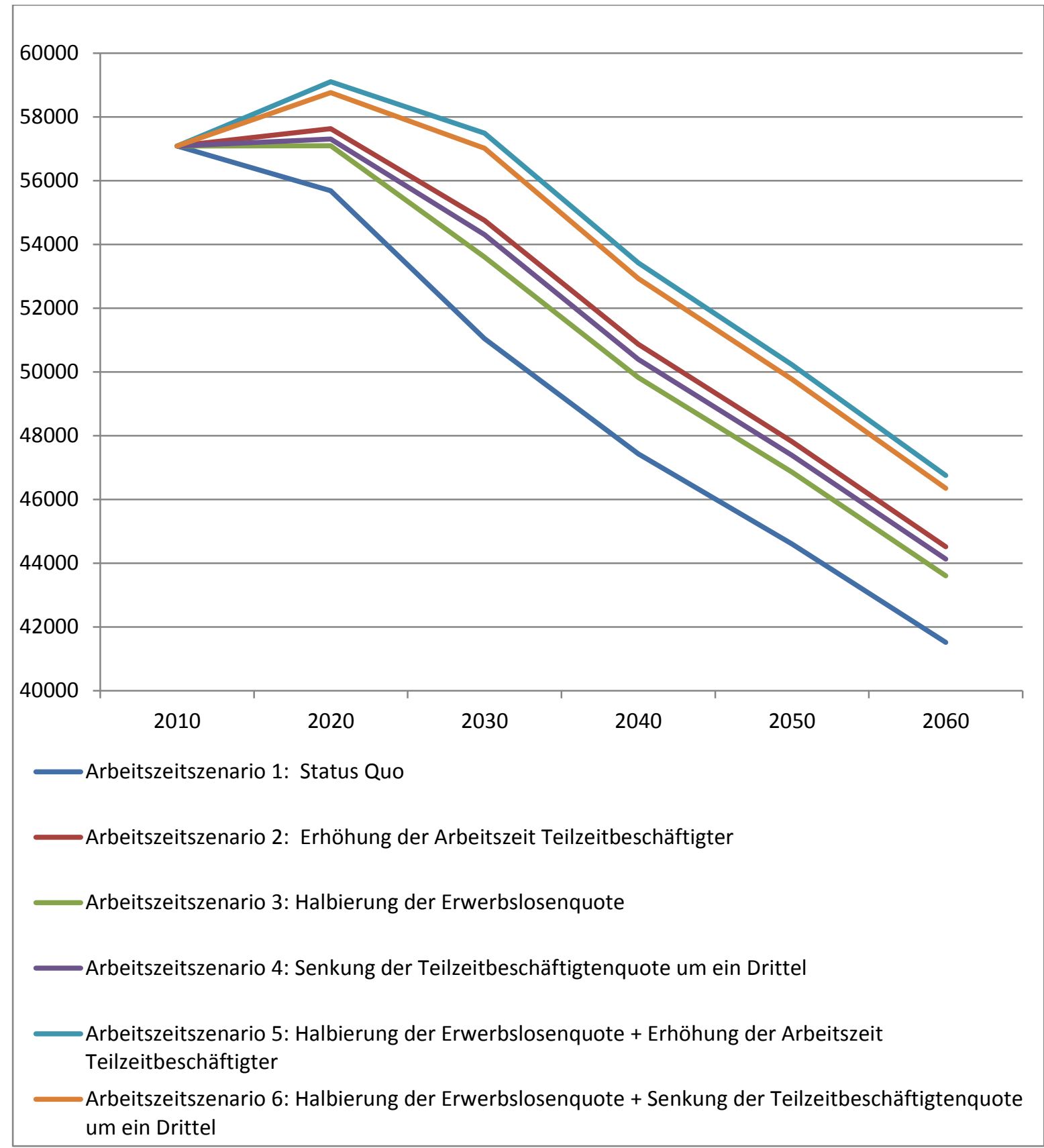

Quelle: Ehing und Moog (2013) 
Beschäftigungsfähigkeit als Schlüssel

Beschäftigungsfähigkeit ist die zentrale Variable für die zukünftige Entwicklung der Erwerbstätigkeit. Beschäftigungsfähigkeit ist aus zweierlei Hinsicht wichtig. Zum einen geht es hier tatsächlich um die Möglichkeit und die Fähigkeit eine Beschäftigung aufzunehmen oder fortzuführen, gerade auch in Zeiten des permanenten und beschleunigten technologischen und strukturellen Wandels. Auf der anderen Seite ist Beschäftigungsfähigkeit aus Sicht der Unternehmen durchaus eine wesentliche Stellgröße für die Produktivität und auch für die Leistungsfähigkeit im Arbeitsprozess. Wenn man den Begriff der Beschäftigungsfähigkeit ein Stück erweitert, muss man sagen, dass auch die Unternehmen beschäftigungsfähig sein müssen. Sie müssen letztlich die Zeichen der Zeit so weit erkennen, dass sie mit den vorhandenen Potenzialen und Personalressourcen produktiv umgehen können. Erst dann können sie sich unter den veränderten Bedingungen als beschäftigungsfähige und damit wettbewerbsfähige Arbeitgeber präsentieren, die Personal für sich gewinnen und dieses produktiv nutzen, halten und weiterqualifizieren können. Das erfordert auch massive Veränderungen, sowohl im Bereich der Politik und teilweise auch im Bereich der Tarifpolitik, als auch, und vor allem, im Bereich der unternehmerischen Personalpolitik. Beschäftigungsfähigkeit bedeutet ein erfolgreiches Matching zwischen Erwerbstätigen und den verfügbaren Beschäftigungsmöglichkeiten.

\section{Veränderte institutionellen Rahmenbedingungen und ihre Auswirkungen}

Deutschland hatte lange Zeit eine Politik betrieben, die den Arbeitsmarkt eher verkleinert hat und verschiedene Gruppen eher aus dem Arbeitsmarkt fern gehalten hat. Hier sind jedoch in den letzten Jahren einige Rahmenbedingungen deutlich verändert worden und dadurch die Arbeitsangebote erhöht worden. Das gilt im Bereich der Frauen, aber auch im Bereich der Aktivierung der Arbeitslosen und insbesondere auch im Bereich der Älteren. Hier wurde über die letzten 10 bis 15 Jahre massiv die Möglichkeit zum vorzeitigen Renteneintritt zurückgefahren. Das zeigt sich unmittelbar in veränderten Erwerbsquoten bei den Älteren. Schließlich ist auch die Zuwanderungspolitik zu nennen. Auch in diesem Bereich hat Deutschland einiges bewegt. Jedoch waren diese Anpassungen nicht so weitgehend, wie man es sich vielleicht aus Sicht des demographischen Wandels und aus Sicht der Arbeitsmarktpolitik wünschen würde. 
Der Arbeitsmarkt ist inzwischen so flexibel geworden, dass wesentlich mehr Personen überhaupt in Beschäftigung gebracht werden. Auch dieser Punkt trägt dazu bei, die Auswirkungen des demographischen Wandels zu lindern und eben überhaupt Beschäftigung zu schaffen. Es wird Berufserfahrung bzw. Arbeitserfahrung vermittelt, die dann in weitergehende produktive Tätigkeiten münden kann.

Insgesamt steigen die Beschäftigungsquoten der Älteren in Europa - und in Deutschland steigen sie mit am stärksten (Abbildung 3). Die Beschäftigungsquote in Deutschland ist von unter $40 \%$ in 2003 auf über 60\% in 2012 gestiegen. Die Konsequenzen auf die Beschäftigungsquoten der Älteren, welche auf der Frühverrentungspolitik seit den späten 1970er Jahren beruht, sind nun mehr als ausgeglichen. Auch das Erwerbsaustrittsalter rückt in Europa nach hinten und Deutschland befindet sich mit auf dem aufsteigenden Pfad (Abbildung 4). Diese Entwicklung ist ebenfalls hilfreich für die Bewältigung des demographischen Wandels. Auch interessant und zunehmend in Diskussion stehend, ist die Tatsache, dass mehr und mehr Personen über 65 Jahren erwerbstätig sind. Das betrifft sowohl Rentner, die einen Minijob haben, als auch viele höher qualifizierte Selbstständige, die einfach über das klassische Renteneintrittsalter von 65 Jahren hinaus, weiterarbeiten.

Abbildung 3: Erwerbstätigenquoten der 55-64 jährigen

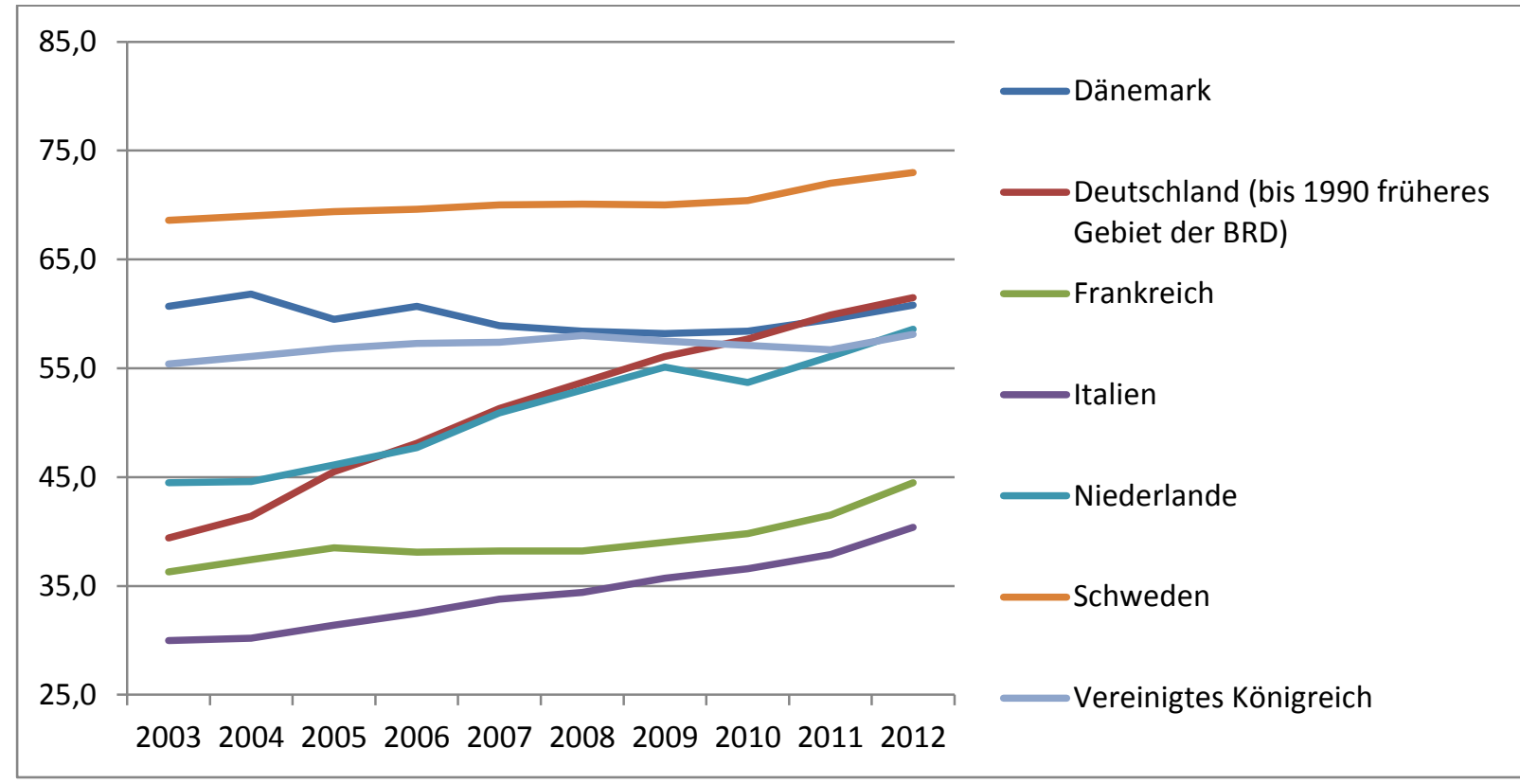


Abbildung 4: Effektives Erwerbsaustrittsalter

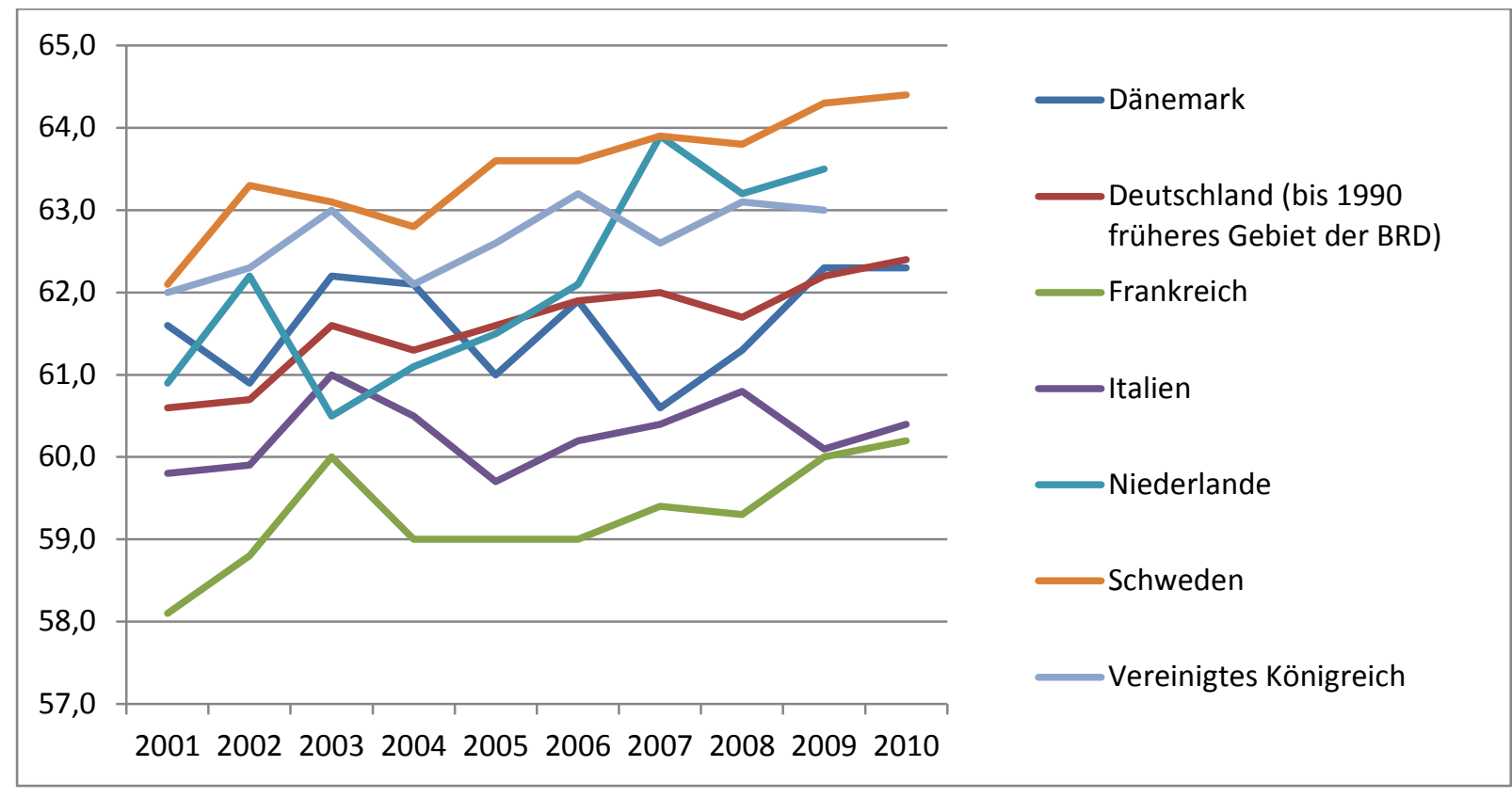

Quelle: Eurostat

Weiterhin gibt es auch noch große Potentiale im Bereich des Beschäftigungsniveaus von geringer Qualifizierten. Diese sind in Deutschland, aber auch in vielen anderen europäischen Staaten, wesentlich weniger stark in den Arbeitsmarkt eingebunden. Hier ist vor allem eine Qualifikationsfrage sicher zu stellen und zu schauen, in wie weit eine stabilere und produktivere Erwerbsbeteiligung genau dieser Personen gewährleistet werden kann. Auch das lebenslange Lernen bietet noch ein weiteres erhebliches Potenzial. Deutschland nimmt in dieser Kategorie keine gute Position ein (Abbildung 5). Weiterbildung ist in Deutschland eher ein Thema für Jüngere und eher höher Qualifizierte. Das bedeutet auch, dass deren Beschäftigungsfähigkeit eher gepflegt und weiterentwickelt wird als die der mittleren und höheren Jahrgänge. Auch bedarf es mehr Bemühungen um die Beschäftigungsfähigkeit jener Personen, die vielleicht nicht zu den besten Leistungsträgern in Betrieben gehören, aber bei denen natürlich auch entsprechende Potenziale vorhanden sind. Gleichzeitig besteht gerade für diese Gruppe ein hohes Risiko, dass der Erwerbsverlauf durch betriebliche Umstrukturierungen, durch Strukturwandel, irgendwann abbrechen kann. Diese Personen haben dann im höheren Lebensalter größere Probleme eine Arbeit zu finden. 
Abbildung 5: Teilnahme an allgemeiner und beruflicher Bildung der 55 bis 64 Jährigen

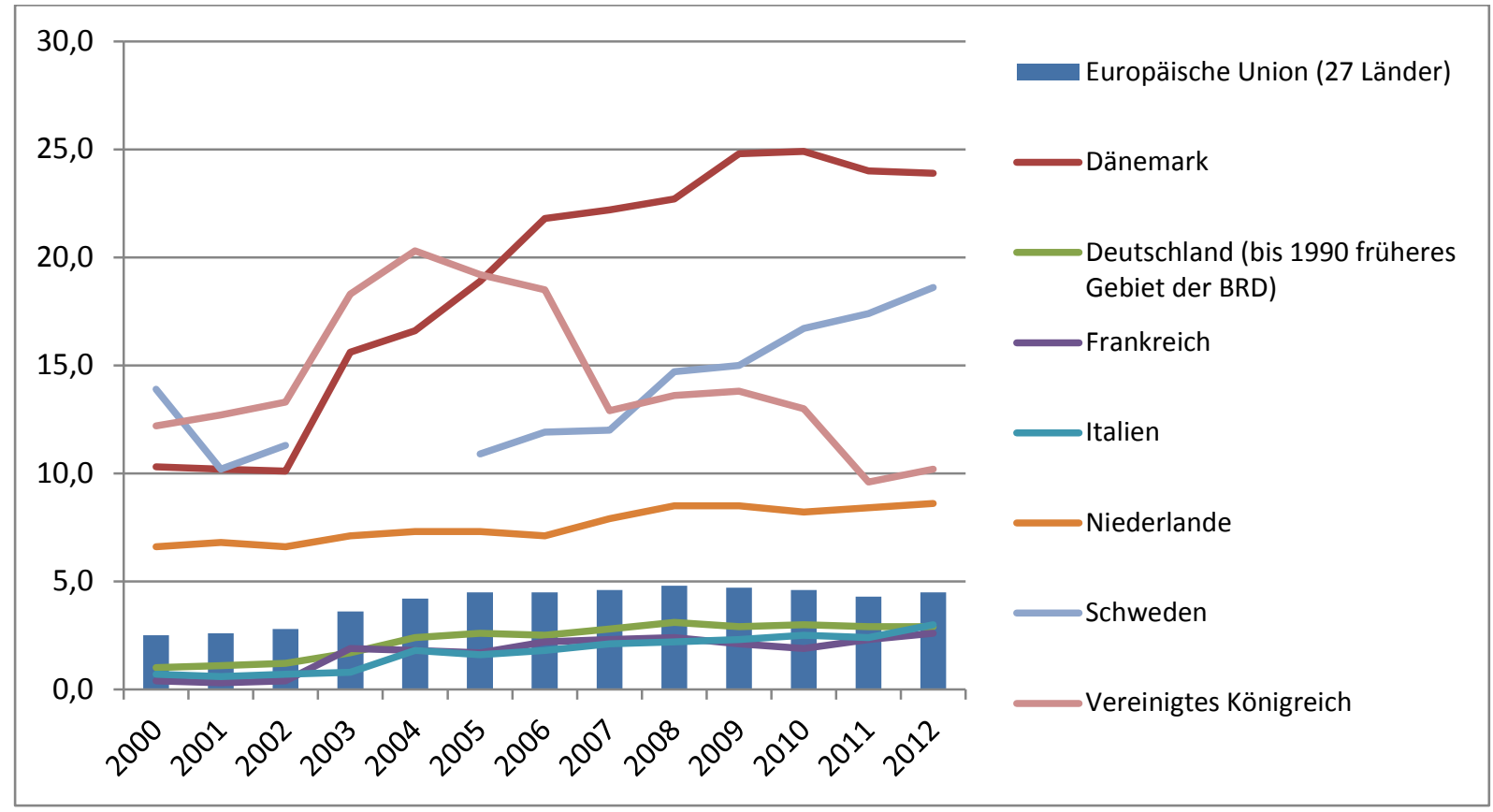

Quelle: Eurostat

Was kann also noch im Bereich der Beschäftigungsfähigkeit politisch, unternehmerisch und auf der individuellen Ebene getan werden? Die Politik hat sicher ständigen Anpassungsbedarf. Hier sind besonders die Ausbildungssysteme zu nennen, die stärker modularisiert werden müssen, um auch im fortschreitenden Lebensalter weitere Qualifikationen zu ermöglichen.

Bereits jetzt ist eine gewisse Tendenz zu beobachten, präventiver an die Arbeitsmarktpolitik heranzugehen. Schwächere Schulabgänger ohne Abschluss oder mit fehlenden Abschlüssen müssen in Zukunft noch effektiver unterstützt werden, um ihnen so den Eintritt in den Arbeitsmarkt zu ermöglichen. Hier tut sich einiges, aber das ist sicher ein Punkt, der noch deutlich an Bedeutung gewinnen muss. Ein Thema, was im Moment noch ein Schattendasein führt, und zwar klar zu Unrecht, ist das Thema systematische Weiterentwicklung im Bereich Weiterbildung. Hier fehlen noch geeignete Kofinanzierungs- und Organisationsmodelle und auch überhaupt besser wirksame Initiativen, um gerade jene Personen in Weiterbildung mit einzubeziehen, die derzeit weder an individuell initiierten noch betriebliche initiierten Weiterbildung teilnehmen, um ihre Beschäftigungsfähigkeit aufrechtzuerhalten. Da damit auch Arbeitslosigkeit vermieden werden kann, ist eine Beteiligung der Arbeitslosenversicherung vertretbar, neben Investitionen der Unternehmen bzw. der Tarifpartner und der beteiligten Individuen. 
Ebenfalls muss das Thema Vermeidung von Erwerbsminderung und frühzeitiger Ausstieg durch Erwerbsminderungsrenten genannt werden. Dies ist vor allem ein Thema unternehmerischer Praxis, aber auch der Politik, die Anreize setzen muss, um entsprechende Probleme erst gar nicht entstehen zu lassen. Und als letzten Punkt der Ausbau von Dienstleistungen, um auch die Beschäftigungsfähigkeit jener Personen zu gewährleisten, die sich eben mit Pflege und mit Kinderbetreuung beschäftigen müssen.

\section{Veränderungen am Arbeitsmarkt und die daraus entstehenden Herausforderungen}

Die Unternehmen müssen jetzt umdenken. Hierbei ist wichtig Beschäftigungsfähigkeit weiter zu definieren und Gruppen prinzipiell als beschäftigungsfähig und auch ausbildungsfähig zu erachten, die bislang oft ein Schattendasein am Arbeitsmarkt geführt haben. Das ist ein Thema, auf das sich die Unternehmen einstellen müssen. Sie müssen bestimmte Routinen und auch Auswahlkriterien hinterfragen und betriebliche Karrieren in Zukunft anders wie bisher gestalten, damit eine längerfristige Bindung erreicht wird.

Vor allem sind Betriebe auch in der Verantwortung wesentlich mehr im Bereich Vereinbarkeit von Familie und Beruf zu tun, um entsprechende Belastungssituationen und Betreuungsengpässe aufzufangen. Das kann in absehbarer Zeit nicht allein durch politische und wohlfahrtsstaatliche Programme aufgefangen werden, sondern die Unternehmen sind hier unmittelbar selbst gefragt. Sie müssen die Beschäftigungsfähigkeit ihrer Angestellten sichern, in dem sie entsprechende Unterstützung organisieren bzw. vermitteln, vielleicht aber auch kofinanzieren.

Im Bereich Produktivität und Beschäftigungsfähigkeit geht es im Wesentlichen darum, die Arbeitswelt deutlich altersgerechter zu gestalten. Schließlich geht die Leistungsfähigkeit nicht zwangsläufig mit dem Alter zurück, sofern entsprechende unterstützende Maßnahmen realisiert werden. Hierbei gibt es zunehmend mehr gute Beispiele, was flexible Arbeitsgestaltung und insbesondere auch, was den Abbau von körperlichen Belastungen im Bereich der verarbeitenden Industrie angeht. Ebenfalls gibt es einige interessante Beispiele aus der Gesundheitspolitik und der Veränderung von technisch belastenden Arbeitsabläufen, wodurch eine längere Beschäftigungsfähigkeit gewährleistet werden kann. Schließlich ist auch der Punkt systematischere Aus- und Weiterbildung entscheidend.

Neben der physischen Belastung der Arbeit ist auch die psychische Belastung, gerade in Bezug auf die Arbeitsverdichtung zunehmend Bestandteil der Diskussion. Heutzutage stellt 
die psychische Belastung eine weitaus größere Gefahr für die Beschäftigungsfähigkeit dar wie die körperliche, welche insbesondere beim verarbeitenden Gewerbe in den letzten Jahrzehnten erfolgreich bekämpft wurde.

Häufige Ursache für eine psychologisch bedingte Erwerbsminderung ist vor allem die Arbeitsverdichtung, aber auch die fehlende soziale Unterstützung am Arbeitsplatz. Bei diesem Thema sind im Wesentlichen auch die Unternehmen gefragt. Übermäßige Belastungen und nicht zu bewältigende Situationen sind zu entschärfen, um ein vorzeitiges Ausscheiden aus dem Erwerbsleben zu vermeiden. Aus der Forschung kennt man einige wesentliche Stellgrößen, die sehr viel zu tun haben mit der betrieblichen Arbeitssituation und dem Betriebsklima und die im Wesentlichen dazu beitragen können, dass solche Situationen nicht entstehen. Das Führungsverhalten, das kollegiale Umfeld und die Arbeitsorganisation müssen in diesem Zusammenhang als eine ganz wesentliche Voraussetzung betrachten werden. Zusätzlich bieten flexible Arbeitszeitmodelle die Möglichkeit die Freizeit und die Arbeitszeit besser in Einklang bringen zu können, wodurch insbesondere außerbetriebliche Spannungsfelder entschärft werden können. Teil dieser präventiven Maßnahmen sollte auch eine entsprechende betriebliche Gesundheitspolitik sein, welche durch Untersuchungen und Beratungen zu einer noch frühzeitigeren Diagnostik beiträgt und im Krankheitsfall zusätzliche Angebote zur Verfügung stellt.

Auch Individuen müssen beschäftigungsfähig bleiben. Aus ihrer Sicht schon, weil das eine ganz wesentliche Voraussetzung ist, um überhaupt an diesem zunehmend auf hohe Produktivität ausgerichteten Erwerbsleben erfolgreich teilhaben zu können. Die Anforderungen an die Individuen steigen tendenziell und es gibt eine gewisse Polarisierung auf dem Arbeitsmarkt. Auf der einen Seite haben die Hochqualifizierten mehr Gestaltungsmöglichkeiten, aber auch zunehmend mehr Gestaltungsverantwortung. Sie müssen mehr Eigenverantwortung übernehmen und sich dem, vielleicht etwas stressigeren und dynamischeren Arbeitsabläufen stellen. Das hat zur Folge, das psychische Dispositionen und auch die Fähigkeit zum Selbstmanagement an Relevanz gewinnen, um selbst den Überblick zu bewahren und die Beschäftigungsfähigkeit für längere Zeit sicherzustellen. Ein anderer Punkt ist sicher auch die Bereitschaft sich zu verändern und am lebenslangen Lernen teilzuhaben. Bei jenen, die am lebenslangen Lernen entweder aus individuellem Antrieb oder auch betrieblicher Initiative nicht so sehr partizipieren, sind durchaus auch Tarifpartner und die Politik gefragt. In diesem Fall müssen sie etwas für die Beschäftigungsfähigkeit tun, um das Risiko von Arbeitslosigkeit oder auch das Risiko von gering entlohnter und auch instabiler 
Beschäftigung zu vermeiden. Diese wichtigen Punkte sollen uns aber nicht davon abhalten insgesamt ein eher positives und optimistisches Bild des demographischen Wandels und auch des Wandels der Arbeitswelt zu entwerfen.

\section{$\underline{\text { Fazit }}$}

Im Wesentlichen sind die Ressourcen vorhanden, um den demographischen Wandel erfolgreich bewältigen zu können - sie müssen nur noch besser genutzt werden. Das ist vielleicht leichter gesagt als getan, weil es nämlich massive Veränderungen auf der Ebene der Unternehmen, auf der Ebene der gesamtgesellschaftlichen Politik und auch auf der Ebene der Sozialpartner und der Tarifpartner impliziert. Die derzeitige Situation ist jedenfalls noch keine, um zu sagen, jetzt sind alle Weichen richtig gestellt und man kann den demographischen Wandel gelassen entgegen sehen. Die staatlichen Rahmensetzungen waren in den letzten Jahren durchaus günstig - aber die neuen rentenpolitischen Entscheidungen stellen einen klaren Fehler dar. Das wesentliche Innovationspotential befindet sich gleichwohl auf der Ebene der Unternehmen. Die größeren und die weiter vorausschauenden Arbeitgeber haben hier schon einiges an Initiativen gestartet. Teilweise auch in Tarifverträgen, wenn man beispielweise an den Bereich der Metallindustrie oder Chemieindustrie oder die Bahn denkt. Dort sind inzwischen auch Demographie-Tarifverträge in Kraft. Es tut sich also schon einiges, aber viele Unternehmen haben das Thema noch nicht systematisch erkannt. Sobald jedoch konkreter Handlungsdruck entsteht, wenn eben keine Fachkräfte mehr gewonnen werden können, wenn es tatsächlich knapp wird, dann werden die Unternehmer ganz massiv Anreize haben zu reagieren. Besser ist es natürlich, rechtzeitig an dieser Stelle anzusetzen, weil dies für Unternehmen in der Wettbewerbssituation mit anderen, sowohl auf der Produktionsseite, als auch auf der Seite der Personalgewinnung, klare Vorteile hat. Interessante Vorbilder sind auf jeden Fall schon heute sowohl in Deutschland, als auch im europäischen Umfeld vorhanden. 
Literatur:

Ehing, D. und S. Moog (2013): „Erwerbspersonen- und Arbeitsvolumenprojektionen bis ins Jahr 2060“, Journal for Labour Market Research, 46(2), 167-182.

Klös, H-P., L. Bellmann, E. Bomsdorf, D. Ehing, W. Eichhorst, S. Moog, M. Schuster (2013): „Arbeitsmarkt“, erschienen in: Rump, J. und N. Walter (Hrsg.): Arbeitswelt 2030 - Trends, Prognosen, Gestaltungsmöglichkeiten, Schäffer Poeschel Verlag, Stuttgart, 33-54. 